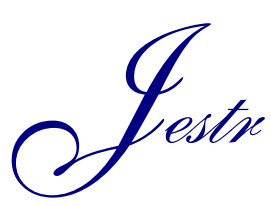

Research Article

\title{
A Developed Design to Enlarge the Spectrum for Analog Electronic Stethoscope
}

\author{
Trinh Quang Duc ${ }^{1, *}$, Nguyen Van Son ${ }^{2}$ and Nguyen Hoai Giang ${ }^{2}$ \\ ${ }^{I}$ Dept. of Biomedical Engineering, Hanoi University of Science and Technology, Hanoi, Vietnam \\ ${ }^{2}$ Faculty of Electronic Telecommunication, Hanoi Open University, Hanoi, Vietnam
}

Received 5 March 2019; Accepted 3 May 2019

\begin{abstract}
Bioacoustic signal from human organ in range of infrasound to ultrasound was not investigated. The signal might provide significant for clinical diagnostics in the future. In this report, a front-end analog system included large spectrum prepolarized condenser microphone sensor and analog amplifier with 4th order active filters, was designed. The measurement spectrums are ranged from $5 \mathrm{~Hz}$ to $40 \mathrm{kHz}$. The amplifier gain can be adjusted in range of $40 \mathrm{~dB}$ to $100 \mathrm{~dB}$. To record the measured data for analysis, the analog signal were converted to numerical data and transfer to computer through a commercial ADC module. To evaluate the system, human heart sound from a volunteer were measured and analyzed with Fourier Transform to obtain signal to noise ratio (SNR) of $50 \mathrm{~dB}$. With the enlargement of the signal spectrum and high SNR as well as high amplification, this pilot device is believed to be the new utility for clinical diagnosis
\end{abstract}

Keywords:bioacoustic signal, infrasound, ultrasound, analog system.

\section{Introduction}

Electronic stethoscope has been produced as commercial products to replace the conventional stethoscope [1] and growing as an advanced medical utility for clinical diagnosis [2]. The interested spectra are $0-2 \mathrm{kHz}$ for tracheal, $0-600$ $\mathrm{Hz}$ for chest wall, 200-800 Hz for bronchial obstruction, 7$75 \mathrm{~Hz}$ for heart, and 100-1000 Hz for bowel [3]. Those sounds are standard records for clinical diagnosis and used as typical medical tools so far. However, in abnormal schemes, the bioacoustic signals may include the range of infrasound or ultrasound as some kinds of animal do [4]. The electronic stethoscope currently uses microphone diaphragm, piezoelectric accelerometers as the sensor to convert the sound intensities to the electrical signal [5]. Both of them has some limitation i.e., piezoelectric sensor devices produce some movement artifacts because of the contact measurement while diaphragm microphones require unique design to eliminate the acoustic noise. Overcome the limitation, Gordon et. al designed a stethoscope which the spectrum ranged in 0.7 to $5000 \mathrm{~Hz}$, with the amplification gain of $15 \mathrm{~dB}$ [10]. On the other invention, Quamar et. al has granted a patent with his device to allow the record of phonocardiography with spectrum ranged in $0.03 \mathrm{~Hz}$ to $1000 \mathrm{~Hz}$ [11]. However, in an investigation from Michael, the range of infrasound dominated in human organs is considerably from $5 \mathrm{~Hz}$ [12]. In addition, because of the nonlinear of human tissues which has the role of sound propagation environment, the high-order harmonic waves is produced. With the limitation of $20 \mathrm{KHz}$, the second order harmonic waves of $40 \mathrm{kHz}$ might occurred. To observe the full spectrum, in this paper, we propose a development to enlarge the spectrum of human bioacoustic signals with a high sensitivity piezoelectric sensor.

*E-mail address: duc.trinhquang@hust.edu.vn ISSN: $1791-2377$ @ 2019 Eastern Macedonia and Thrace Institute of Technology. All rights reserved. doi:10.25103/jestr.122.24

\section{Methods \& Design}

The system was designed with a bell and an active filter which the function diagram is shown in the Figure 1. Since the sound measured from human body is very weak, the signal should be amplified with a bandpass filter so called active filter ranged from $5 \mathrm{~Hz}$ to $40 \mathrm{kHz}$ to observe even the signal from infrasound to ultrasound. The range is chosen because almost of ultrasound produced from biological systems is not over $40 \mathrm{kHz}$ [4].

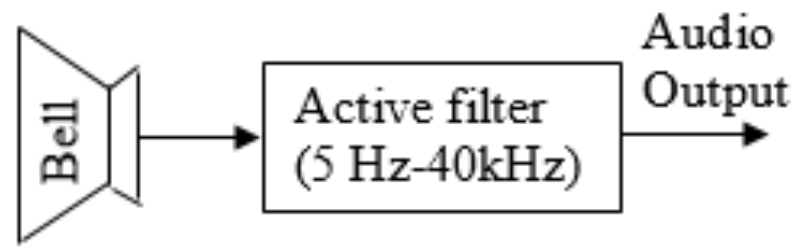

Fig. 1. The system function diagram

To obtain the acoustic signal in the wide spectrum range, a bell is designed as the format of traditional diaphragm stethoscopes with a piezoelectric transducer is set at the bottom of the bell. The sound signal is acoustically amplified in the bell then converted to the electrical signal through the transducer. The mechanical structure of the bell has advantage to minimize the environment noise, hence, increase the signal to noise ratio (SNR) of the measure signal. The mechanical diagram of the bell design is shown in Fig. 2 , where the diaphragm is designed as a drum truncated cone with base diameter is $43 \mathrm{~mm}$, top diameter is $17 \mathrm{~mm}$, and height of $16.5 \mathrm{~mm}$. On the top of the drum truncated cone, a drum cylinder with the outer diameter of the top cone has height of $12 \mathrm{~mm}$ is added to hold the piezoelectric transducer. The bell is made by aluminum alloy in thickness of $1 \mathrm{~mm}$ with the tolerance of $0.25 \mathrm{~mm}$. 


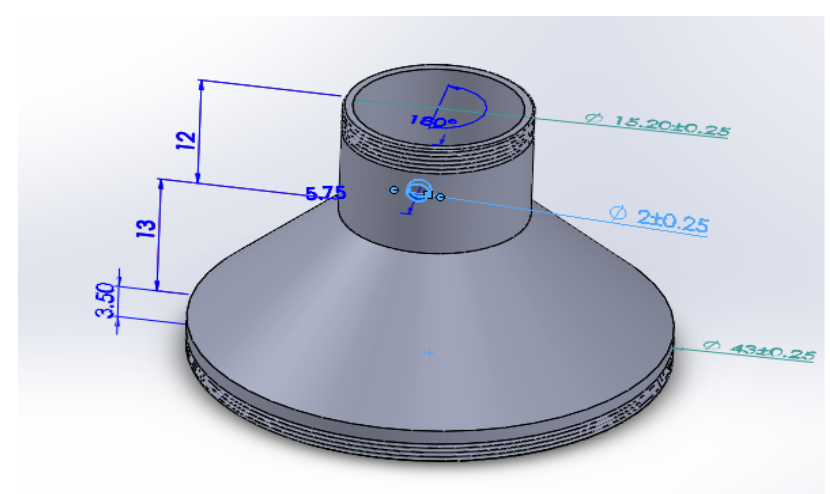

Fig 2. The mechanical diagram of the bell design

The amplitude of the bioacoustic signal directly output from the bell is very weak, typically in range of $10^{-3}$ to $10^{-5}$ $\mathrm{Pa}$ [6], therefore, the measured signal should be amplified then filtered in a specified spectrum depending the sound characteristics of each organs to increase the SNR. In this design, the amplifiers and the bandpass filters are combined to be an active filter. The active bandpass filter is separated as an active highpass filter and an active lowpass filter, which the diagram is shown in Fig. 3

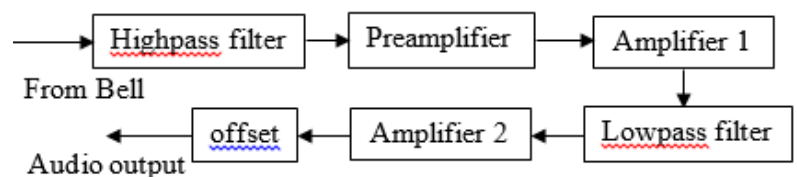

Fig. 3. The diagram of the active filter

The highpass filter diagram is presented in Fig. 4. As the figure shown, the circuit is designed as passive filter with the cut-frequency is set at $5 \mathrm{~Hz}$ through the value of the capacitor $\mathrm{C} 1$ and $\mathrm{R} 1$ as $470 \mathrm{nF}$ and $68 k \Omega$, respectively.

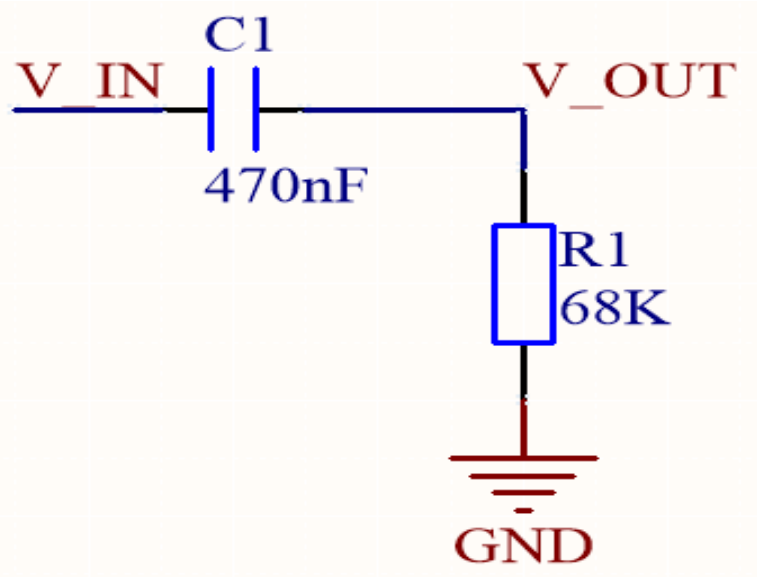

Fig. 4. The circuit diagram of the highpass filter.

For the assessment, the frequency response is measured in comparison with a numerical simulation computed by a commercial software named as Proteus v.7.8. The measured data is collected with an experiment in which the input frequencies value is varied in range of 0 to $1 \mathrm{kHz}$ with a step of $5 \mathrm{~Hz}$ while the output amplitude values are measured. The experimental processes are performed with the hardware modelled as ELVIS-II and the driving software from $\mathrm{Na}$ tional Instrument (NI). The measure result is shown in Fig. 5 indicated the cut-frequency litter bit higher (about $5.5 \mathrm{~Hz}$ ) comparing to the numerical simulation.

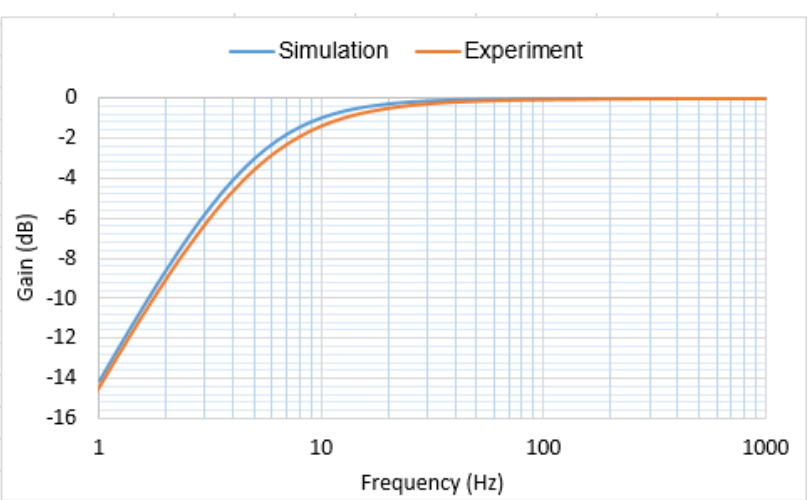

Fig. 5. The frequency response of the passive highpass filter including the simulation and the experiment data.

Since the amplitude of the output signal should be ranged from 0 to $5 \mathrm{~V}$ to adapt with the fix reference voltage of a regular ADC (Analog to Digital Converter) module while the input signal in range of few ten microvolts, the total amplifier is estimated as $100 \mathrm{~dB}$. To reduce the distortion and increase the SNR, the active filter is designed as 3 stages in which the first stage is the preamplifier. The preamplifier is designed as the circuit which the schematic is showing in Fig. 6 .

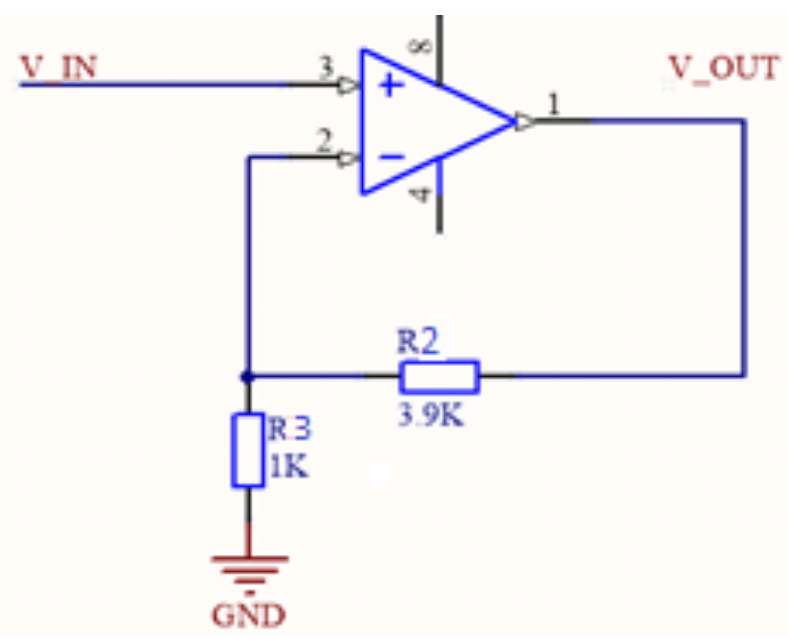

Fig. 6. The schematics of the preamplifier circuit.

The gain amplitude ratio of the preamplifier is set as 5 with $\mathrm{R} 2$ and $\mathrm{R} 3$ are $3.9 k \Omega$ and $1 k \Omega$, respectively. The output signal from the preamplifier is fed into amplifier 1, which the circuit schematic is shown in Fig. 7. The signal is amplified though an inverted amplifier circuit with the gain possibly adjusted by a variable resistor. Since the loudness of the each human organ provides the measured signal is very difference, the gain value is adjusted to adapt with the specific measure range. In this circuit, the values of the resistor were set as showing in the Fig. 7, the R4, R5 are fixed resistor and has value of $1 k \Omega$, the VR1 can adjust value in range of 0 to $40 \mathrm{k} \Omega$. The gain amplitude ratio can be adjusted from 2 to 50 in theoretical calculation. The values are shown in Tab. 1.

Because of the wide spectrum in the measure range, the linearity of the frequency response is needed to be determined to demand the precision of the measurement. To determine the frequency response, the input sinusoidal waves ranged from 0 to $80 \mathrm{kHz}$ in step of $5 \mathrm{~Hz}$ were used as input signal and the measurement from the output amplitude values are recorded. The procedure of the experiment is performed with ELVIS-II and a simple program written in 
Labview v.2013. As the result, the frequency responses are shown in the Fig. 8, in which, the responses with 8 gain values corresponding to the theoretical values were investigated shows the high linearity in the range of 0 to $50 \mathrm{kHz}$, demanded the desire measurable range. The performance of the amplifier indicates the reliable for the practical measurement, hence, the circuit also was applied to the amplifier 2.

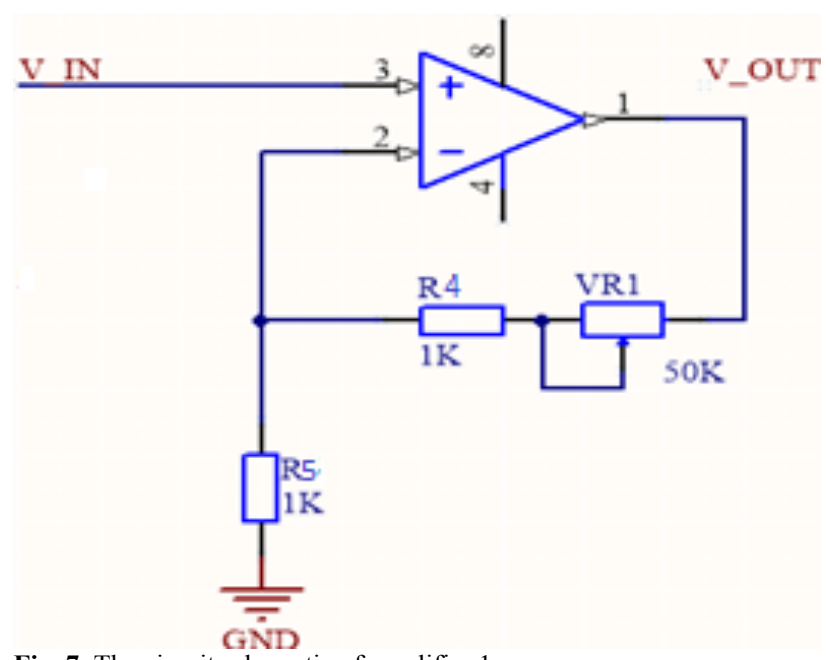

Fig. 7. The circuit schematic of amplifier 1.

Table 1. The gain amplitude ratio values of amplifier 1 in theoretical calculation.

\begin{tabular}{c|c|c}
\hline $\mathbf{V R} 1$ & $\mathbf{K}$ & Gain (dB) \\
\hline $4 \mathrm{~K} \Omega$ & 5 & 13,9794 \\
$9 \mathrm{~K} \Omega$ & 10 & 20 \\
$14 \mathrm{~K} \Omega$ & 15 & 23,52183 \\
$19 \mathrm{~K} \Omega$ & 20 & 26,0206 \\
$24 \mathrm{~K} \Omega$ & 25 & 27,9588 \\
$29 \mathrm{~K} \Omega$ & 30 & 29,54243 \\
$34 \mathrm{~K} \Omega$ & 35 & 30,88136 \\
$39 \mathrm{~K} \Omega$ & 40 & 32,0412 \\
\hline
\end{tabular}

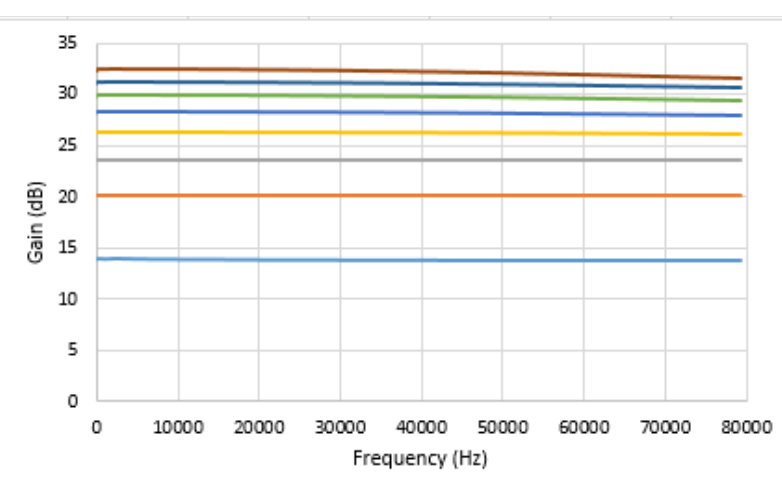

Fig. 8. The practical investigation of the amplifier frequency responses.

To reduce the noise from high frequency, between the stages of the amplifiers, an active lowpass filter $4^{\text {th }}$ order was designed as shown in the Fig. 9, where resistors R6, R7 have the same value of $2.2 \mathrm{k} \Omega$, the value of resistor R8, R9 is 3.9 $k \Omega$, and the capacitors value of $\mathrm{C} 1, \mathrm{C} 2, \mathrm{C} 3, \mathrm{C} 4$ is chosen as $0.001 \mathrm{uF}$. The cut frequency is set at $40 \mathrm{kHz}$ with the values of the capacitors and the resistors are indicated in the figure. Practically, the frequency response also investigated with the same method applied to the experiments for the highpass filter and the amplifier 1. The response is shown in the Fig. 10. As the figure showing, the cut frequency of the filter is set at $30 \mathrm{kHz}$, little bit lower in comparison with the simulation response. However, at $40 \mathrm{kHz}$, the gain reaches to $-7 \mathrm{~dB}$ while the calculation cut frequency located at $-5 \mathrm{~dB}$, not too high in the pass range of the measurement spectrum.

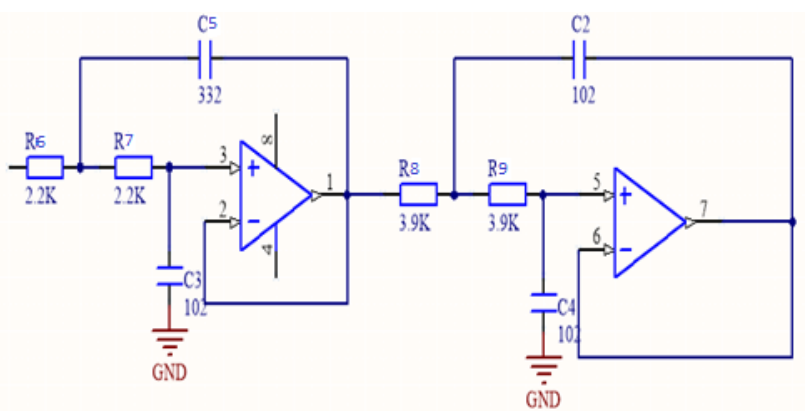

Fig. 9. The schematic circuit of the active lowpass filter.

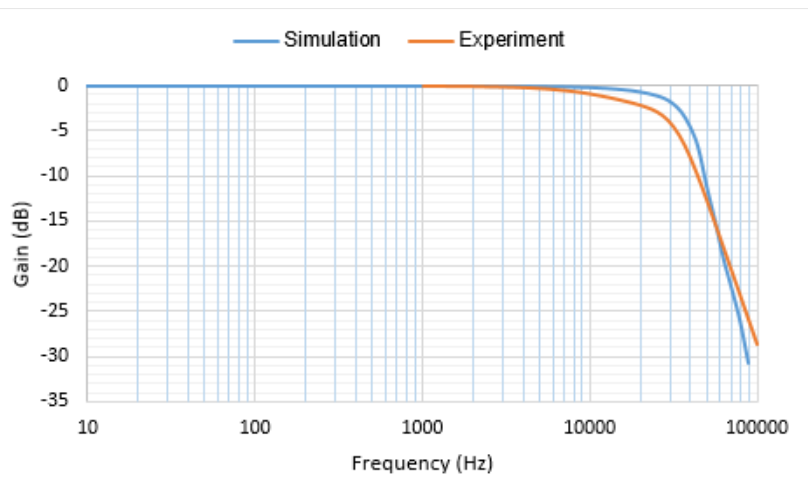

Fig. 10. The comparison of the theoretical and experimental frequency responses of the active lowpass filter.

Because of the electrical signal from the bell generated by piezoelectric sensor under micro mechanical vibration include positive and negative value in comparison with the ground pole, a symmetrical power source was used for the amplifiers. For this purpose, the symmetrical power source of $+9 \mathrm{~V}$ and $-9 \mathrm{~V}$ with virtual ground was used. However, the input range of the $\mathrm{ADC}$ is 0 to $5 \mathrm{~V}$ as requirement. Therefore, the output signal should be offset to adjust the peak to peak amplitude of the signal to the range of positive voltage. The offset circuit was designed based on the added circuit using operation amplifier, which the schematic is shown in Fig. 11. For simplicity the values of R10 to R13 is set equally is $10 \mathrm{k} \Omega$. V_offset can be simply adjusted by a potentiometer circuit.

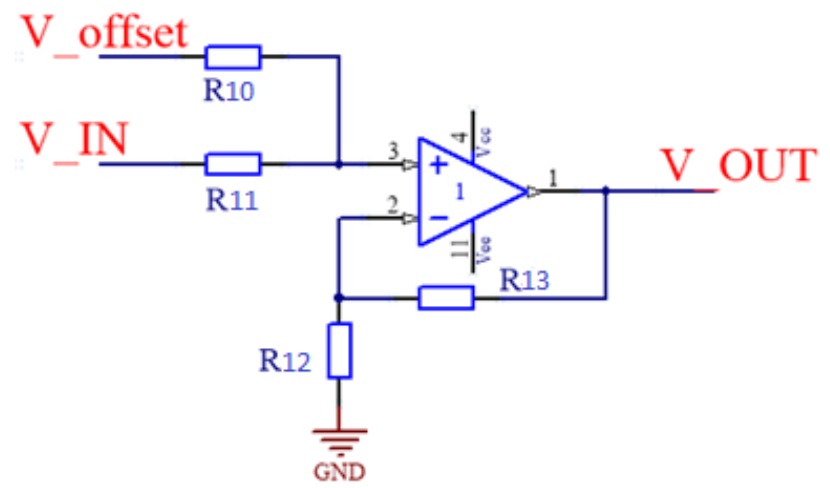

Fig. 11. The schematic of the offset circuit.

The experimental investigation as well as the theoretical simulation indicated the analog circuit design system is high performance to demand the requirement of the bioacoustics signal measurement. 


\section{Materials}

To convert the bioacoustic signal to the electrical signal, in this development, the pre-polarized microphone which is a type of condenser using diaphragm with high sensitivity was used. The sensor named as QTC40 produced by Earthworks Inc. company, whose the frequency response spectrum is shown in Fig. 12.

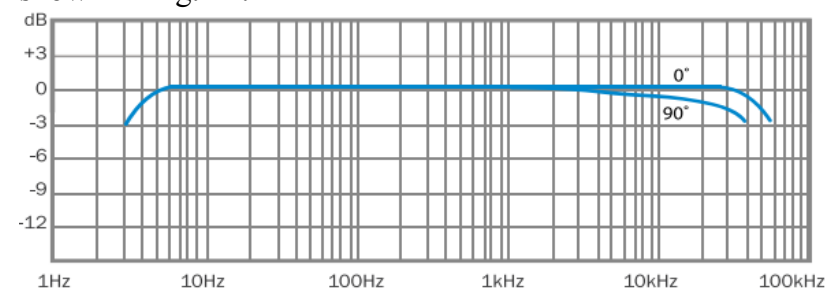

Fig. 12. The frequency response spectrum of QTC40 sensor [8].

As the figure show, the sensitivity of the sensor is flatten in range of $5 \mathrm{~Hz}$ to $40 \mathrm{kHz}$. With the measured range from 5 $\mathrm{Hz}$ to $40 \mathrm{kHz}$ to collect the bioacoustic signal data, this specification is demanded the requirements. To transfer the sound from body to the diaphragm and reduced the reflection from the acoustic impedance mismatch, a silicone rubber with the acoustic impedance is equivalent to distilled water was used.

For the amplification, the operational amplifier (OPAM) is the main factor effecting to the quality of the collected signal as well as the improvement of the SNR. In this design, the OPAM of OPA134 was used. With the high performance in gain stability as show in Fig. 13 and the CMR (Common-Mode Rejection) characteristics indicated in Fig. 14 , the OPAM demands the requirement of amplification with high gain, low distortion, and low noise in wide spectrum range.

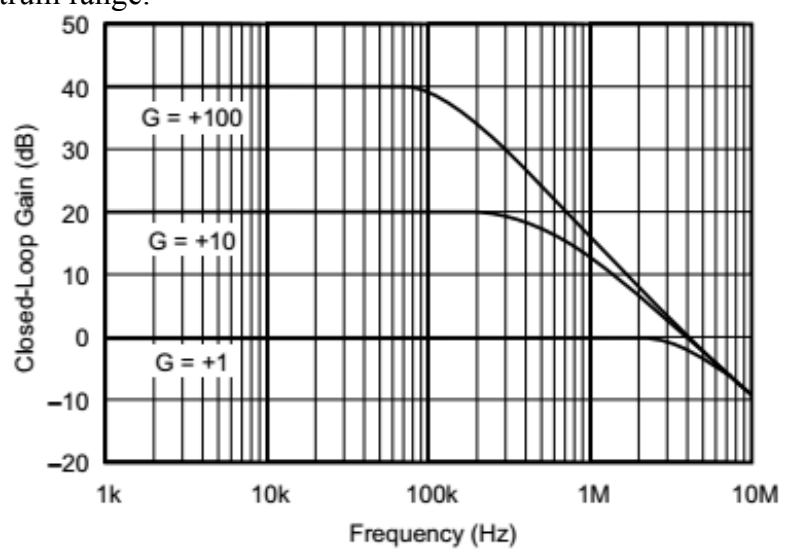

Fig. 13. The gain vs. frequency response of OPA134 [9].

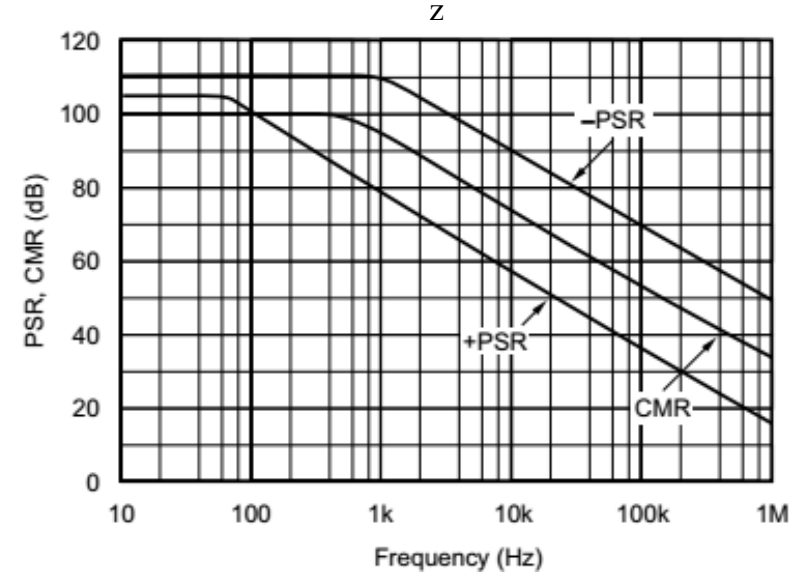

Fig. 14. The CMR vs Frequency response of OPA134 [9].
As the performance of the crucial devices, the design theoretically demand the measurement of human organ bioacoustic signal.

\section{Results}

As the preliminary result, the circuit is fabricated as shown in the Fig. 15 with 3 OPA134 and 2 knob of variable transistor for gain adjustment. For the experiment, the output of this device is fed into a commercial module made for PC interface named as Arduino. Based on the ADC module, the signal are recorded with a sampling frequency of $100 \mathrm{kHz}$ through a software designed in Labview. Because of the numerical data after convert from the electrical signal, the signal analysis algorithm possibly applied to assert the bioacoustic signal.

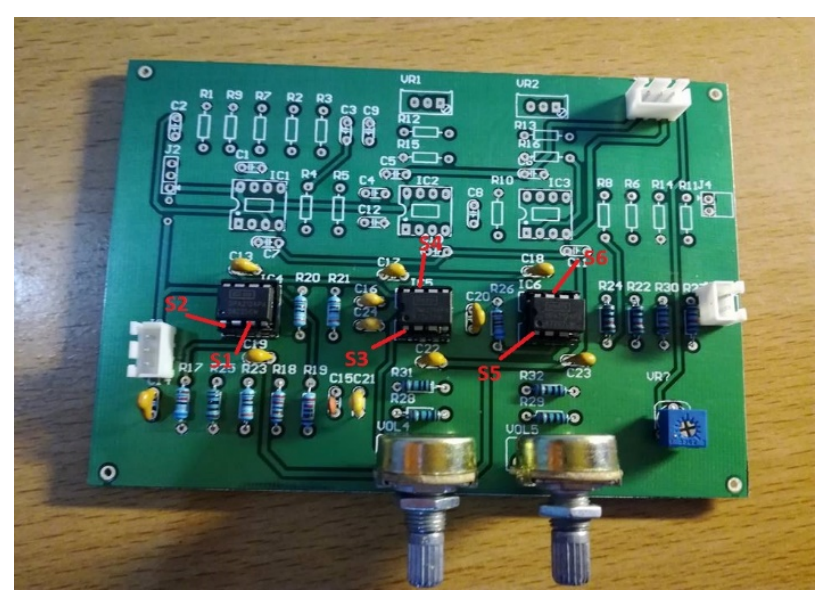

Fig. 15. The circuit of the measurement device.

Figure 16 shows the bioacoustic signal of a human heart was measured from a volunteer at the output point of the highpass filter. As the figure shown, the peaks occurred in the signal corresponds to the heart-beat rhythm. The peak-topeak amplitude reaches to $0.03 \mathrm{~V}$.

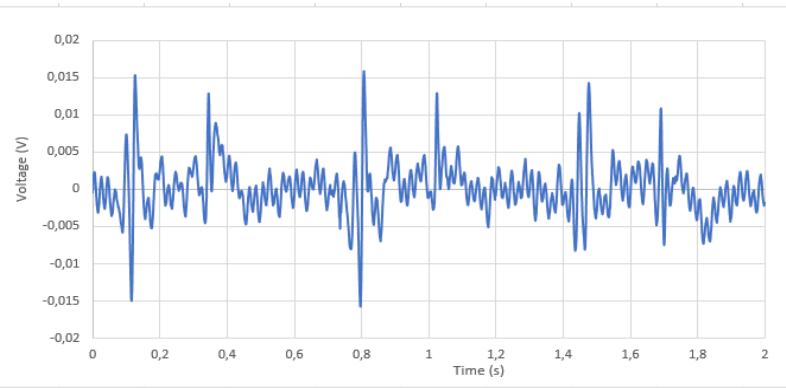

Fig. 16. The heart-beat signal from output of the highpass filter.

The signal, after amplified through Preamp module, is greater as shown in Fig. 17. The peak-to-peak amplitude of the signal reaches to $0.15 \mathrm{~V}$. This signal, in diagram of the circuit, is fed into the lowpass filter. The result shows in the Fig. 18.

The signal, after filtered through the lowpass filter is almost the same as the signal output from Preamp module. The result also indicated that the high frequency noise is not effect to this system.

Figure 19 and 20 shows the signal after amplified through Amplifier 1 and Amplifier 2, respectively. After amplified through Amplifier 1, the peak-to-peak signal reaches to $0.8 \mathrm{~V}$ and $4.5 \mathrm{~V}$ through Amplifier 2. The signal 
through Amplifier 2 is considered as the output signal of the analog system because the offset circuit has no effects to the signal but adjust the zero point of the measured values.

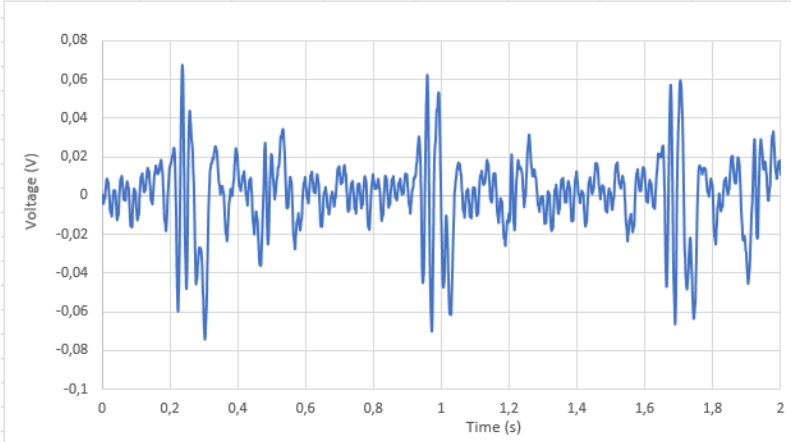

Fig. 17. The signal from the hear-beat after amplified through Preamp.

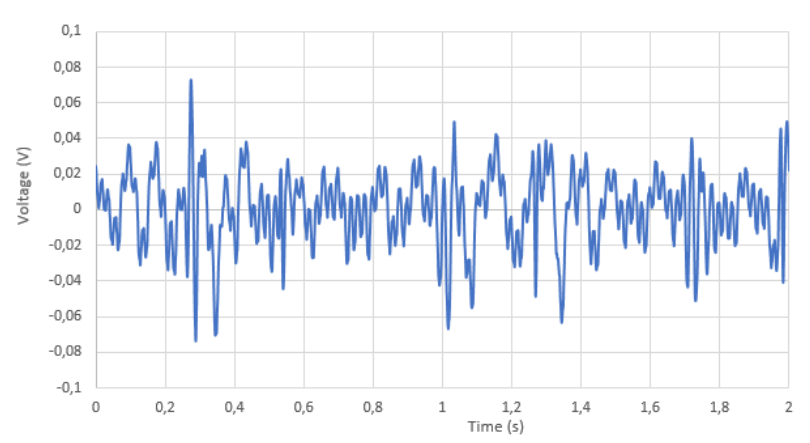

Fig. 18. The signal after filtered through the lowpass filter.

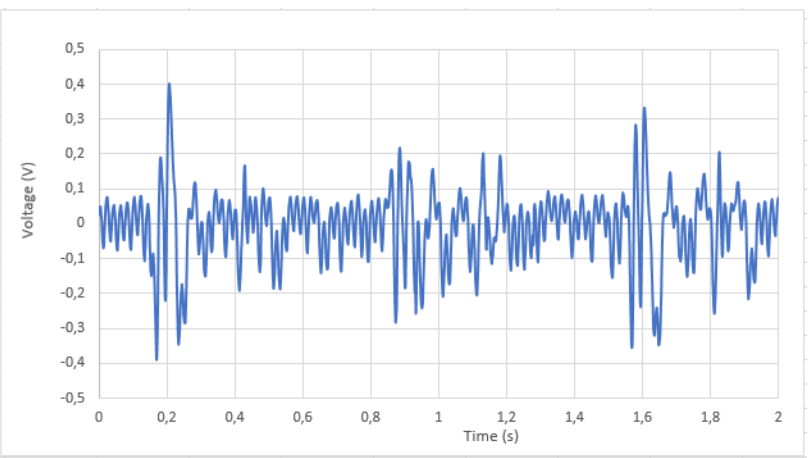

Fig. 19. The output signal from Amplifier 1

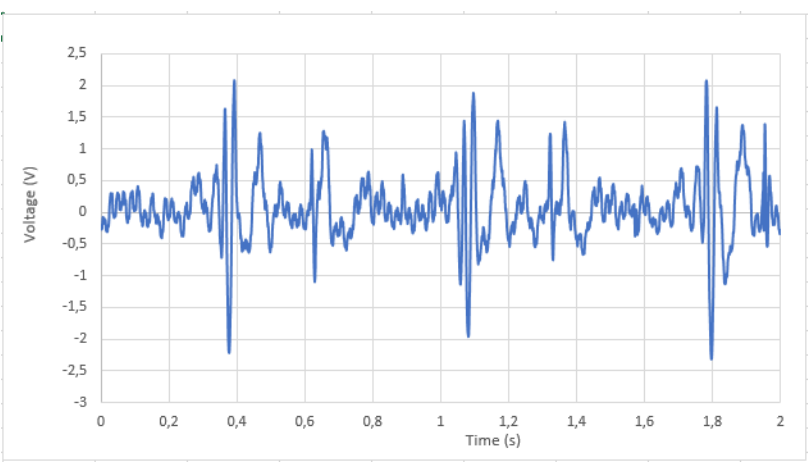

Fig. 20. The output signal from Amplifier 2.
To assert the SNR of the measured signal the data analyzed with simple algorithm of Fourier Transform. The measured data which presented from the Fig. 16 to Fig. 20 to show the performance of the system, is transferred to frequency domain as show in the Fig. 21

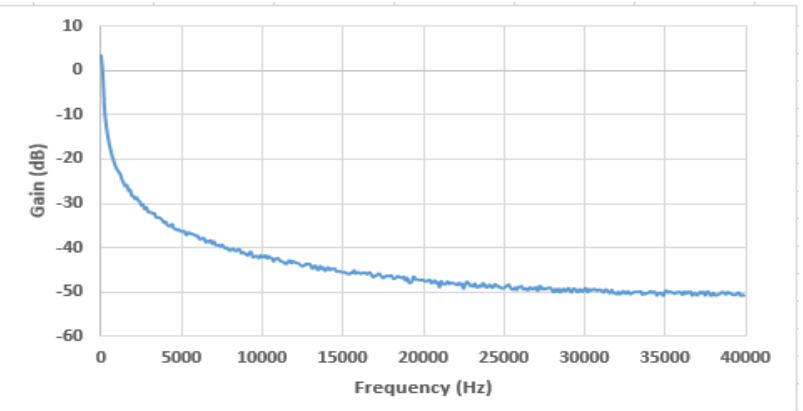

Fig. 21. The spectrum of system output signal.

The experiments prove the capability and performance of the analog system. With the high gain amplification, low distortion and ground noise, the analog system possibly apply to a series of experimental measurement to collect the crucial signal for clinical diagnosis.

\section{Conclusion}

An analog for bioacoustic signal measurement in wide spectrum range was designed. The gain of the amplification possibly reaches to $100 \mathrm{~dB}$ with the SNR obtained up to 50 $\mathrm{dB}$. With the wide range spectrum flatten from $5 \mathrm{~Hz}$ to 40 $\mathrm{kHz}$ and the specification of designed amplification, the system allows data collection of the bioacoustic signal from infrasound to low-frequency ultrasound.

With these system performance, this device believed to be useful tool for bioacoustic signal acquisition experiments even infrasound and/or ultrasound range which the current technologies are considering to measure in the auditable range. The bioacoustic signal also believed to be the new utilities for clinical diagnosis.

For the near future, based on this pilot device, an acquisition of data from patients in hospitals will be planned. The number of the data samples can be used to investigate the roles of infrasound and ultrasound from human organs in clinical diagnosis.

\section{Acknowledgment}

This work is financially supported by the program named as B2017-MHN.01 of Vietnam Ministry of Education and Trainning.

This is an Open Access article distributed under the terms of the Creative Commons Attribution License

\section{References}

1. GosReports. Global Stethoscopes Market Research Report 2016, December 30, 2016.
2. Global Industry Analysts, Inc. Advanced Electronic Innovations to Benefit Global Sales of the Iconic Stethoscopes Which Continue to Remain Indispensable to Medical Practice. MCP-3363, April 2017. 
Trinh Quang Duc, Nguyen Van Son and Nguyen Hoai Giang/Journal of Engineering Science and Technology Review 12 (2) (2019) 179 - 184

3. Hadjileontiadis, L. J., Rekanos, I. T. and Panas, "Bioacoustic Signals", Wiley Encyclopedia of Biomedical Engineering, 6 Vol. Set, pp. 329 - 334, S. M. 2006.

4. Fletcher, Neville H, "Animal Bioacoustics, Chapter 17 in Springer Handbook of Acoustics", pp. 821-841, Springer, 2007.

5. L. Vannuccini, J. E. Earis, P. Helisto“, B. M. G. Cheetham, M. Rossi, A. R. A. Sovija“rvi, and J. Vanderschoot, "Capturing and preprocessing of respiratory sounds.", Eur. Respir. Rev., 10(77):616-620. 2000.

6. Chengyu Liu, David Springer, Qiao Li, Benjamin Moody, Ricardo Abad Juan, Francisco J Chorro, Francisco Castells, José Millet Roig, Ikaro Silva, Alistair E W Johnson, Zeeshan Syed,Samuel E Schmidt, Chrysa D Papadaniil, Leontios Hadjileontiadis, Hosein Naseri, Ali Moukadem,Alain Dieterlen, Christian Brandt, Hong Tang, Maryam Samieinasab, Mohammad Reza Samieinasab, Reza Sameni, Roger G Mark and Gari D Clifford, "An open access database for the evaluation of heart sound algorithms.", Physiological Measurement, 37 (12), 2016.
7. Kawai, Heiji (1969). "The Piezoelectricity of Poly (vinylidene Fluoride)". Japanese Journal of Applied Physics. 8 (7): 975

8. Earthworks, "Omni High Definition Microphone for Quiet Sources”, QCT40 User's Guide, June 2014.

9. Texas Instruments, "High Performance Audio Operational Amplifiers" OPAx134 datasheet, Dec. 1997 [Revised Oct. 2015].

10. Gordon ES, Lagerwerff JM, "Electronic stethoscope with frequency shaping and infrasonic recording capabilities", Aviat Space Environ Med. 47(3):312-6, 1976

11. Qamar A. Shams, Allan J. Zuckerwar, Albert L. Dimarcantonio, "Infrasonic stethoscope for monitoring physiological processes", Patent No. 10092269, October 9, 2018.

12. Michael A. Persinger, "Infrasound, human health, and adaptation: an integrative overview of recondite hazards in a complex environment”, Nat Hazards, 70:501-525, 2014 